\title{
Few-second EELS mapping with atomic-resolution
}

\author{
Berit Goodge and Lena Kourkoutis
}

\section{School of Applied and Engineering Physics, Cornell University, United States}

The complex oxides offer a wide variety of tunable functional properties from multiferroicity to superconductivity governed by the subtle interplay between local lattice and electronic degrees of freedom. While bulk techniques such as diffraction refinements and spectroscopic measurements can probe these responses with very high precision, atomic-resolution STEM and EELS can directly probe the real-space correlation between these effects. Furthermore, advanced in situ experiments offer the potential to explore not only static coexistence of states, but also to track the dynamic evolution of competing phases across transitions or under the application of external stimuli. How does a metal to insulator transition manifest at the nanoscale? What are the structural and electronic responses and how are they linked? While developments in STEM instrumentation and data processing have widened the experimentally accessible phase space with in situ heating, cooling, electrical biasing, and mechanical straining capabilities, the reduced stability of many of these techniques limits the practical time scales for high-resolution experiments. Certain methods, such as STEM imaging, have overcome many of these challenges by greatly reducing the collection times and averaging several consecutive image frames. In situ spectroscopic studies, however, have thus far been greatly limited by comparison due to the much longer characteristic acquisition times. A typical atomic-resolution STEM image may be acquired in less than one second, while an atomic-resolution EELS map often requires several minutes due to the much longer pixel times involved (milli- vs. microseconds). From a materials standpoint, forgoing the detailed chemical and electronic characterization that EELS can offer leaves an often important part of the problem unexplored. To advance these proposed correlative high-resolution studies of functional materials or devices, the implementation of the STEM-EELS experiment - including the relevant instrumentation and hardware - must be updated for orders-of-magnitude decreases in acquisition time.

Ultimately, the goal for high-resolution in situ EELS is to reduce total acquisition time without sacrificing the quality of each recorded spectrum. To date, atomic-resolution in situ cryo-EELS mapping has been demonstrated, made possible in large part by using an EELS detector with reduced noise [1] and increased readout speed [2,3]. Improvements to other parts of the STEM-EELS instrument have opened additional opportunities: for example, brighter sources can greatly increase the probe current and thus the total signal in a single spectrum of fixed acquisition time [4]. Figure 1a shows single core-loss La-M $\mathbf{M}_{4,5}$ spectra acquired with different source + detector configurations: a 300 kV FEI Titan Themis X-FEG with Gatan UltraScan1000 CCD (red) and Gatan K2 direct detector (blue), and a TFS Spectra X-CFEG at $120 \mathrm{kV}$ with Gatan Continuum scintillator CMOS camera (green). At short $2.5 \mathrm{~ms}$ acquisition times, the benefits of low detector noise and improved point spread function between the CCD (red) and direct detector (blue) are clear. Further improvement can be obtained by moving to a high-brightness cold field emission source. In probes of comparable size $(\sim 1 \AA)$, the X-CFEG can operate with five times greater probe current and three times narrower energy spread, such that the per-spectrum acquisition time can be reduced by more than 10 to $0.2 \mathrm{~ms}$ and preserve similar SNR at the same energy dispersion $(0.5 \mathrm{eV} / \mathrm{ch})$ on the scintillator CMOS (green). Chemical analysis can be aided by averaging spectra from a single atomic column, typically less than 100 pixels. If the noise in each spectrum is sufficiently uncorrelated, such averaging will improve the SNR thereby enabling more robust data analysis and interpretation. Correlations in the noise will sum additively at a similar rate to the real signal and give rise to false patterns in the spectrum which could obscure true features in the EELS fine structure. We assess the noise correlations for the scintillator CMOS detector by measuring the Pearson correlations between 100 spectra recorded with the beam blanked (Fig. 1c) with a correlation value of 0 implying perfectly random noise, while 1 is perfectly correlated. The average correlation for this detector with a high-quality dark reference applied at the acquisition is very low (0.005) which is also reflected in the 
SNR improvements by summing individual core-loss spectra (Fig. 1b). This combination of a low-noise detector and high-brightness source is therefore well-poised for rapid in situ spectroscopic experiments.

Harnessing the high-brightness X-CFEG as well as the fast readout of the Continuum GIF and scintillator CMOS detector, we demonstrate atomic-resolution EELS mapping of $\mathrm{TbScO}_{3}$ using a $450 \mathrm{pA}$ probe with perpixel dwell times of only $0.2 \mathrm{~ms}$, an order-of-magnitude increase from previous results [2,3]. With a total spectrum image frame time of 7 seconds, the structural symmetries of the A- and B-site sublattices are preserved even in the elemental map, as highlighted by the presence (lack) of superlattice peaks in the $\mathrm{Tb}-\mathrm{M}_{4,5}$ $\left(\mathrm{Sc}-\mathrm{L}_{2,3}\right)$ map FFTs. This demonstration paves the way for in situ experiments in which highly localized spectroscopic measurements can be correlated directly to a specific lattice response.

Supported by NSF DMR-1539918, DMR-1429155, DMR-1719875.
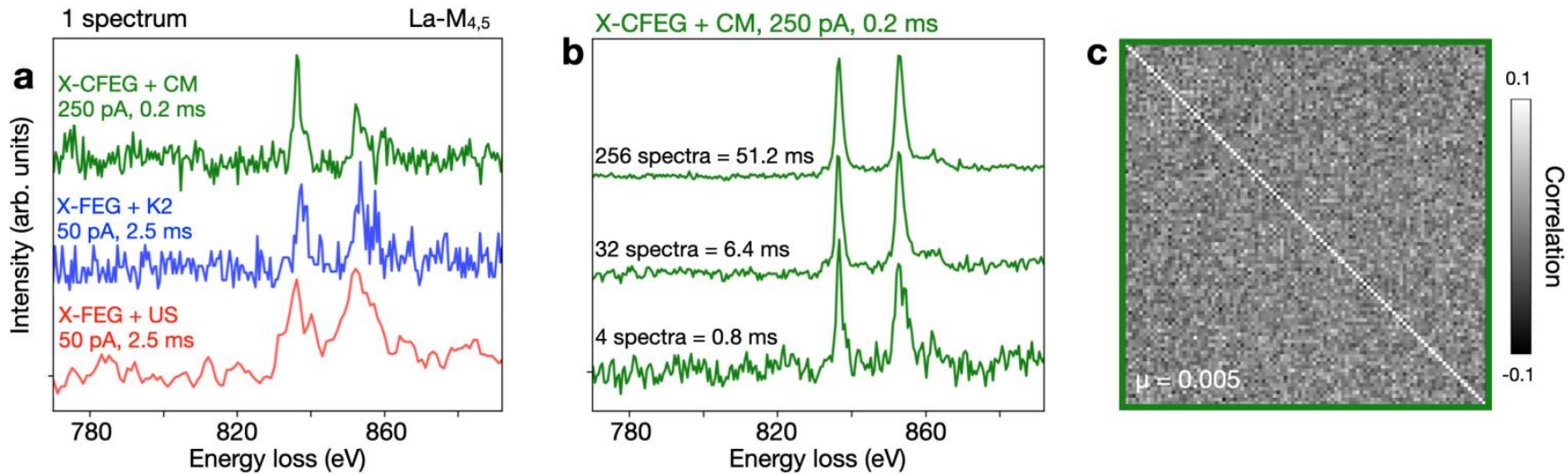

Figure 1. (a) Short dwell time core-loss spectra demonstrate the performances of different source and detector combinations. The $300 \mathrm{kV} \mathrm{X-FEG} \mathrm{(red,} \mathrm{blue)} \mathrm{has} \mathrm{an} \mathrm{energy} \mathrm{spread} \mathrm{of} \sim 0.9 \mathrm{eV}$ and probe current of $\sim 50 \mathrm{pA}$. The $120 \mathrm{kV}$ X-CFEG (green) has an energy spread of $\sim 0.35 \mathrm{eV}$ and probe current of $250 \mathrm{pA}$. The improved energy resolution and increased probe current enable spectra with similar SNR to be acquired on the scintillator CMOS camera in only $0.2 \mathrm{~ms}$, less than one-tenth the $0.25 \mathrm{~ms}$ exposure time used with the X-FEG+K2 combination. (b) The SNR can be further improved by averaging spectra from multiple pixels. For atomicresolution EELS mapping, each atomic column is typically 16-64 pixels. (c) Correlation matrix of 100 spectrum readouts (beam blanked) on the scintillator CMOS detector. The Pearson correlation coefficients for spectra $\mathrm{i}, \mathrm{j}$ are shown in the corresponding matrix entry (diagonal entries are 1 and have been neglected in the reported average).
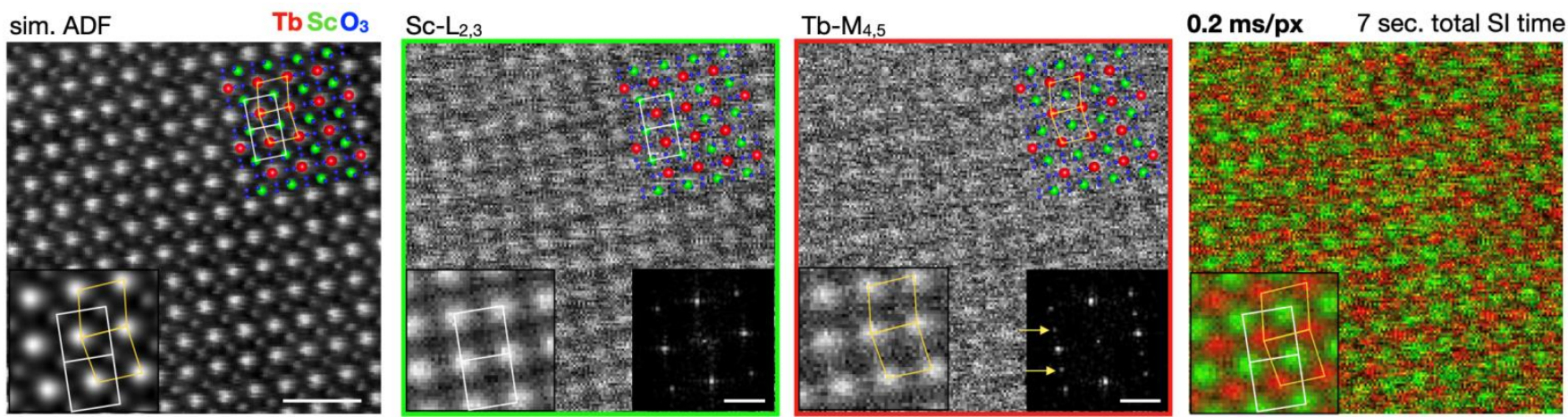

Figure 2. Atomic-resolution EELS maps of $\mathrm{TbScO} 3$ acquired with the Spectra X-CFEG and Continuum $\mathrm{GIF} /$ camera at $200 \mathrm{kV}$ with a $0.2 \mathrm{~ms} / \mathrm{px}$ dwell time. The 7 second total SI time preserves the orthorhombic distortions of the lattice, reflected in the appearance of [001/2] superlattice peaks (yellow arrows) in the FFT of 
the Tb elemental map. Bottom left insets show unit cell averages from each image ( 1.9 nm fields of view). Scale bars $1 \mathrm{~nm}$ (image) and $4 \AA-1$ (FFTs).

\section{References}

[1] Hart, et al. Sci. Rep. 7 (2017), p. 8243.

[2] Goodge, et al. (2020) arXiv: 2007.09747.

[3] Goodge, et al. Microsc. \& Microan. S1 (2018).

[4] Goodge, et al. Microsc. \& Microan. S2 (2020). 